\section{Motivación y emoción en música. \\ Estado de la cuestión y aportaciones para la innovación educativa}

Motivation and Emotion in Music. State of the art and contributions for educational innovation

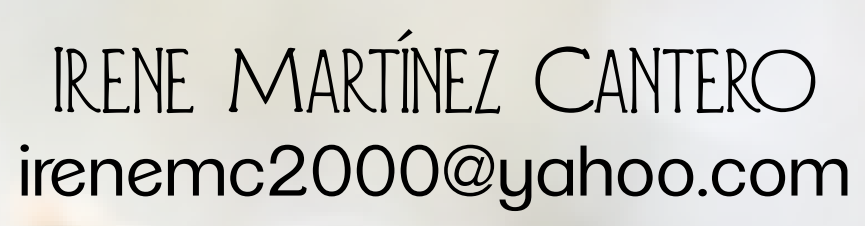

Conservatorio Profesional de Música de Pilar de la Horadada $\rightarrow$ Recibido 26/06/2017

$\checkmark$ Aceptado 15/09/2017

\section{Resumen}

Aunque la Educación Musical presenta un campo reducido de estudios de investigación, los aportes de las últimas décadas parecen indicar un cambio significativo en relación al interés que están desentrañando, así como en lo que se refiere a los temas que son objeto de estudio en ella. En este artículo se presenta una aproximación al conocimiento educativo sobre Motivación y Música que se ha ido aportando a lo largo del tiempo en diferentes países, acercándonos para ello también y desde la Neurociencia al cercano constructo de Emoción y Música. Se concreta todo ello en el campo de la Piscología de la Música como disciplina que está comenzando la andadura hacia su consideración como campo propio y diferenciado de conocimiento. Así mismo, desde estas aportaciones generales y particulares, se presentan propuestas para la innovación que pueden ayudar al docente en Música en cualquiera de sus ámbitos y niveles.

\section{Palabras clave}

Música · Motivación · Emoción · Educación · Innovación 


\section{Introducción}

Los temas de estudio que ha abarcado la investigación sobre enseñanza y aprendizaje en música, sin duda alguna, y como bien afirma Sloboda (2012), han sufrido un cambio y un progreso considerable. Desde los años 70 y 80 del siglo pasado hasta la actualidad, estos temas han estado centrados según la década en la que nos detengamos en aspectos como procesos básicos, desarrollo de habilidades musicales, música real en la actualidad o motivación y emoción. A estos temas se une una perspectiva crítica del fenómeno educativo en música que pueden englobarse en la desmitificación del concepto de talento y la consideración de lo emocional, junto a lo motivacional, y no sólo las habilidades técnicas, como componentes ya más que esenciales en el ámbito pedagógico. Pero, además, incluso el tratamiento de los mismos ha sufrido transformaciones en disciplinas que, desde el respaldo de la Psicología, han encontrado en la multidisciplinariedad y las metodologías cualitativas nuevos caminos antes impensables (Casas-Mas, 2008).

Desde el punto de vista etimológico, la palabra motivación tiene su origen en el verbo latino "movere", que significa mover. Es, por tanto, lo que mueve y está vinculada a la acción física y mental (Achtziger, \& Collwitzer 2008), puesto que impulsa y guía la acción; esto es, da la energía para actuar y hace que la acción se encamine hacia una meta (McClelland, 1985; Elliot, 2008; Thrash, \& Hurst, 2008). Por lo tanto, el comportamiento es un buen indicador para medir la motivación (Reeve, 1992; Montero \& Huertas, 2003; Heckhausen, \& Heckhausen, 2008) y algunas de sus medidas son la preferencia o elección entre alternativas, la latencia del comportamiento ante el estímulo, el esfuerzo o aplicación de recursos físicos y cognitivos para el desarrollo de la tarea, la persistencia en ella y los indicadores expresivos de placer o displacer de las emociones que acompañan las acciones (Rodríguez, 2009). Si consideramos al organismo como un todo, muchas veces resulta difícil establecer diferencias entre los términos de activación, motivación y emoción (Hernández-Pina, 2001).

\section{Key words}

Music $\cdot$ Motivation $\cdot$ Emotion $\cdot$ Education $\cdot$ Innovation 
Sin embargo, aunque la capacidad de expresión de la música genera ciertas concordancias para todas las personas y algunos parámetros de control han quedado trazados (Juslin, 1996, 1997a, 1997b, 1997c), la naturaleza exacta de lo que puede y no puede ser expresado aún no se ha comprendido en su totalidad (Collier, 2007). Además, se sabe que la percepción es biocultural y que depende de estímulos físicos y sensaciones involucrados al mismo tiempo que de su selección y organización (Vargas, 1994). Es por todo ello que Motivación, Emoción y Música resultan un punto de encuentro para la mayor parte de las investigaciones relacionadas con la Psicología de la Música que posibilitan establecer un marco del estado de la cuestión general aplicable a casi todos los estudios de investigación en Educación Musical.

\section{Marco Teórico}

En el campo específico de la Música son ya algunas las investigaciones sobre Motivación en su estudio. En general, estas pueden catalogarse atendiendo a cuatro criterios: motivación en el inicio de los estudios musicales, motivación en el inicio de los estudios instrumentales, importancia de la motivación y relevancia del contexto (Tabla 1).

Estas investigaciones aportan perspectivas diversas pero enlazadas. Así, en relación al inicio de los estudios musicales, algunas lo relacionan con los entornos personales cercanos al alumnado, tales como familia o grupo de pares (Hallam, 2001, 2002; Kinney, 2010; Cremades, Herrera, \& Lorenzo, 2011) o en su capacidad de conexión con las emociones personales (StGeorge, Holbrook, \& Cantwell, 2014). Para el inicio de los estudios instrumentales suelen vincularse con el género del estudiante o con los conocimientos previos y pautas sociales (Hallam, Rogers, \& Creech, 2008; Herrera, \& Cre$\propto$ mades, 2011; Vasil, 2013; Herrera, \& Oya, 2014; McPherson, Osborne, Barrett, Davidson, \& Faulkner, 2015) siendo un factor importante para predecir el éxito o fracaso de los estudios musicales, así como el mejor pronóstico para la práctica individual (Hallam, 2002; Millican, 2016).
Regulación y motivación son aspectos relacionados de la práctica musical, ya que hay relación entre autorregulación y uso de estrategias de estudio, y entre motivación y esfuerzo en el estudio y desarrollo de habilidad musical. Los estudiantes que disfrutan cuando estudian, con un enfoque productivo y que usan recursos sociales y físicos, se esfuerzan más y tienen mayores comportamientos de regulación (Austin, \& Haefner, 2006). Existen vínculos entre motivación intrínseca y comportamiento autorregulado en la práctica instrumental, ya que, además del tiempo de estudio, es importante el tipo de estrategias que se utiliza, siendo esencial la percepción de autonomía del alumnado (Renwick, 2008; Hallam, 2013). Por otra parte, el nivel de experiencia tiene relación con las subescalas de motivación: vida social e importancia de la interpretación, satisfacción con el rendimiento, autocreencia en la habilidad y disfrute con las actividades instrumentales. Estas subescalas junto con apoyo social y afirmación en conjuntos predicen las aspiraciones de los estudiantes (Hallam, Creech, Pageorgi, Gomes, Rinta, Varvarigou, \& Lanipekun, 2017).

El contexto del alumnado resulta, por tanto, otro de los aspectos de mayor relevancia cuando indagamos el constructo motivación en música. Así, la sensación de apoyo de otras personas y la valoración práctica de los estudios musicales parecen predecir altos niveles de integración y actitudes positivas hacia situaciones de aprendizaje (Maclntyre, Potter, \& Burns, 2012). Sin embargo, la percepción personal del alumnado es distinta, tal y como muestra Ghazali (2006), que aporta que los niños entienden el aprendizaje musical como una globalidad de lo formal y lo informal, lo cual influye positivamente en la importancia que otorgan a éste. Es en este punto donde consideramos que debe indagarse un poco más para establecer relaciones sociales también con Emoción en Música que puedan vincularse a la motivación hacia ella para la innovación educativa. 


\begin{tabular}{|c|c|c|}
\hline Temática & Referencia & Aportaciones \\
\hline \multirow{4}{*}{$\begin{array}{l}\text { Motivación } \\
\text { en el inicio } \\
\text { de los } \\
\text { estudios } \\
\text { musicales }\end{array}$} & Hallam (2002) & $\begin{array}{l}\text { Si hay las posibilidades de estudio en el centro, los alumnos se encuentran muy influenciados por el colegio, la familia, los amigos y por intérpretes famosos. El interés } \\
\text { por la música o el querer aprender y llegar lejos son minoritarios }\end{array}$ \\
\hline & Kinney (2010) & $\begin{array}{l}\text { El logro académico futuro y la estructura familiar biparental predicen la decisión de participación en una banda de música. Éstos mismos, junto con el nivel económico } \\
\text { alto o el género femenino son también significativos para la continuación en la agrupación }\end{array}$ \\
\hline & Cremades, Herrera, y Lorenzo (2011) & $\begin{array}{l}\text { Son los padres los que ejercen mayor influencia, aunque también lo hacen hermanos, primos y amigos. Son escasas las opciones relativas al aprendizaje musical o al } \\
\text { gusto por la actividad }\end{array}$ \\
\hline & StGeorge, Holbrook, y Cantwell, (2014) & La conexión que la música establece con las emociones humanas tiene importancia en el inicio y continuación con los estudios \\
\hline \multirow{5}{*}{$\begin{array}{l}\text { Motivación } \\
\text { en el inicio } \\
\text { de los } \\
\text { estudios } \\
\text { istrumentales }\end{array}$} & Hallam, Rogers, y Creech (2008) & $\begin{array}{l}\text { Existen patrones de instrumentos en padres e hijos en relación a estereotipos de género. Otros factores que influyen en la elección instrumental son individuales (edad, } \\
\text { timbre, géneros musicales, forma de tocar, símbolos de identidad y persistencia), sociales (cultura, religión, expectativas relacionadas con estereotipos, profesor, padres, } \\
\text { iguales y hermanos) y propios del instrumento (posibilidad, coste, transporte, apariencia, timbre, tesitura, familia, forma de interpretación y tipo de repertorio) }\end{array}$ \\
\hline & Herrera, y Oya (2014) & $\begin{array}{l}\text { La elección instrumental tiene gran influencia en el correcto desarrollo de los estudios musicales y en el abandono de los mismos, siendo los factores que la determinan: } \\
\text { las opciones posibles tras la prueba de aptitud, el conocimiento previo de instrumentos, el género y la edad (por la lista de orden para elección de instrumento) }\end{array}$ \\
\hline & Vasil (2013) & Los factores extrínsecos que influyen en el comienzo y continuación del aprendizaje instrumental son la familia y el entorno, así como factores sociales y económicos \\
\hline & $\begin{array}{l}\text { McPherson, Osborne, Barrett, David- } \\
\text { son, y Faulkner (2015) }\end{array}$ & A la mayor parte de los estudiantes de estudios generales les gustaría aprender a tocar un instrumento extraescolarmente \\
\hline & Millican (2016) & $\begin{array}{l}\text { Es importante que los profesores ayuden a los estudiantes en la elección instrumental. La motivación para tocar un instrumento en concreto resulta un factor importante } \\
\text { para predecir el éxito y la persistencia de los estudios }\end{array}$ \\
\hline \multirow{12}{*}{$\begin{array}{l}\text { Importancia } \\
\text { de la } \\
\text { motivación }\end{array}$} & Hallam (2001) & $\begin{array}{l}\text { En los primeros cursos el estudio individual de los alumnos, si es continuo, se debe a presiones y actitudes intervencionistas por parte de los padres. Esto provoca en los } \\
\text { alumnos una visión negativa y desemboca en resistencia al estudio }\end{array}$ \\
\hline & Hallam (2002) & El mejor pronóstico sobre la actitud hacia la práctica es la autodeterminación individual \\
\hline & Ghazali (2006) & $\begin{array}{l}\text { Aunque el aprendizaje musical suele ser poco valorado, los niños que sí lo hacen, es por motivaciones intrínsecas. Se hallan diferencias en esta percepción del aprendizaje } \\
\text { musical relacionadas con la etnia y con el género, siendo más positiva para las niñas }\end{array}$ \\
\hline & Austin, y Haefner (2006) & $\begin{array}{l}\text { Regulación y motivación son aspectos relacionados de la práctica musical, ya que hay relación entre autorregulación y uso de estrategias de estudio, y entre motivación y } \\
\text { esfuerzo en el estudio y desarrollo de habilidad musical. Los estudiantes que disfrutan cuando estudian, con un enfoque productivo y que usan recursos sociales y físicos, } \\
\text { se esfuerzan más y tienen mayores comportamientos de regulación }\end{array}$ \\
\hline & Navas, Iborra, y Sampascual, (2007) & $\begin{array}{l}\text { En el área de música existen tres tipos de metas en este área: metas de aprendizaje, metas de logro y metas de refuerzo social, que coinciden con las de otros aprendizajes } \\
\text { académicos }\end{array}$ \\
\hline & Renwick (2008) & $\begin{array}{l}\text { Existen vínculos entre motivación intrínseca y comportamiento autorregulado en la práctica instrumental. Además del tiempo de estudio, es importante el tipo de estra- } \\
\text { tegias que se utiliza, siendo esencial la percepción de autonomía del alumnado }\end{array}$ \\
\hline & Marco (2010) & $\begin{array}{l}\text { Existe relación a largo plazo entre tipos de metas motivacionales y percepciones de éxito, metas de superación personal y de aprendizaje, autoconcepto académico y } \\
\text { metas de logro, autoconcepto familiar y atribución del alto rendimiento al esfuerzo, y autoconcepto académico y autoconcepto físico }\end{array}$ \\
\hline & Hallam (2013) & $\begin{array}{l}\text { Para el compromiso a largo plazo existen relaciones entre nivel alcanzado y tiempo de estudio, y entre expectativas de futuro y: disfrute con la actividad, actitudes hacia } \\
\text { la interpretación y valor percibido de la música, autopercepción musical y uso de estrategias en el estudio }\end{array}$ \\
\hline & Holgado, Navas, y Marco (2013) & $\begin{array}{l}\text { Se encuentran relaciones entre autoconcepto, atribuciones causales, metas académicas y contenido de las metas de los estudiantes, y entre todas ellas y el rendimiento } \\
\text { académico }\end{array}$ \\
\hline & León, Nuñez, Zuleica, y Bordón (2015) & $\begin{array}{l}\text { Existen dos grupos caracterizados por altas o bajas puntuaciones en todas las variables: motivación intrínseca, pensamiento crítico y rendimiento académico. En ambos } \\
\text { la motivación intrínseca tiene efecto sobre el pensamiento crítico, aunque éste afecta en mayor medida al resultado del grupo de los estudiantes con bajas puntuaciones }\end{array}$ \\
\hline & $\begin{array}{l}\text { McPherson, Osborne, Barrett, David- } \\
\text { son, y Faulkner (2015) }\end{array}$ & $\begin{array}{l}\text { Los alumnos que no estudian música extraescolarmente de mayor edad, otorgan a la música menor competencia, interés, importancia y utilidad, siendo al contrario para } \\
\text { los que estudian música extraescolarmente. Las chicas perciben la música como más importante y útil, pero menos interesante que los chicos }\end{array}$ \\
\hline & $\begin{array}{l}\text { Hallam, Creech, Pageorgi, Gomes, Rin- } \\
\text { ta, Varvarigou, y Lanipekun, (2017) }\end{array}$ & $\begin{array}{l}\text { El nivel de experiencia tiene relación con las subescalas de motivación: vida social e importancia de la interpretación, satisfacción con el rendimiento, autocreencia en } \\
\text { la habilidad y disfrute con las actividades instrumentales. Estas subescalas junto con apoyo social y afirmación en conjunto predicen las aspiraciones de los estudiantes }\end{array}$ \\
\hline
\end{tabular}




\begin{tabular}{|c|c|c|}
\hline \multirow{6}{*}{$\begin{array}{l}\text { Relevancia } \\
\text { del contexto }\end{array}$} & Hallam (2002) & $\begin{array}{l}\text { Cultura, subculturas, sociedad, momento histórico, lugar, instituciones, familia y grupo de pares se relacionan con las características individuales de las personas, ejerci- } \\
\text { endo influencia en la motivación y en el logro }\end{array}$ \\
\hline & Ghazali (2006) & Los niños entienden el aprendizaje musical como una globalidad de lo formal y lo informal, lo cual influye positivamente en la importancia que otorgan a éste \\
\hline & Creech, y Hallam (2009) & $\begin{array}{l}\text { Existe relación entre interacciones entre padres, alumnos y maestros, tipo de participación de los padres y autoeficacia y satisfacción personal. Son destacables en impor- } \\
\text { tancia de influencia sobre el aprendizaje la ambición y satisfacción de los padres, el liderazgo del maestro y la reciprocidad de estas interacciones }\end{array}$ \\
\hline & Herrera, y Cremades (2011) & $\begin{array}{l}\text { El tipo de música que escuchan los estudiantes de conservatorio tiene gran influencia mediática, aunque tienen mayor relevancia en este aspectos las actividades extra- } \\
\text { escolares que las escolares }\end{array}$ \\
\hline & MacIntyre, Potter, y Burns (2012) & $\begin{array}{l}\text { La sensación de apoyo de otras personas y la valoración práctica de los estudios musicales parecen predecir altos niveles de integración y actitudes positivas hacia situ- } \\
\text { aciones de aprendizaje }\end{array}$ \\
\hline & $\begin{array}{l}\text { Hallam, Creech, Pageorgi, Gomes, Rin- } \\
\text { ta, Varvarigou, y Lanipekun (2017) }\end{array}$ & $\begin{array}{l}\text { El nivel de experiencia está relacionado con la motivación por vida social. La motivación por la vida y el apoyo social también predicen las aspiraciones de los estudiantes } \\
\text { de música }\end{array}$ \\
\hline
\end{tabular}

Tabla 1. Aportes de investigaciones afines (elaboración propia)

\section{Contribuciones para la innovación educativa}

Los procesos de innovación educativa generan importantes reacciones emocionales que van a estar directamente relacionadas con las actitudes y la motivación de los estudiantes. Se debe comprender la naturaleza de estas emociones y de sus fuentes para poder diseñar estrategias que faciliten la adaptación del estudiante a su contexto (Candelario, 2009). Así, los estudios sobre motivación en la Psicología General, imponen cada vez más, para una realidad compleja y en cambio continuo, la necesidad de su contemplación desde una concepción holística (Meyer, \&Truner, 2002). De esta forma, se deben considerar a la vez los elementos cognitivos, emocionales, motivacionales y sociales para que puedan conocerse los procesos implicados en las experiencias emocionales, el significado que estas experiencias tienen para las personas implicadas, y el contexto histórico-social donde emergen (Schutz, \& DeCuir, 2002).

Dentro de estos estudios, el comportamiento de los individuos constituye un indicador directo para medir la motivación (Montero, \& Huertas, 2003; Reeve, 1992). Es por tanto, más que adecuado para el profesorado conocer los modos en los que sus alumnos se enfrentan a la actividad musical (Tabla 1) o a la música en general. Con respecto a lo último, Hargreaves (2012) expone su Modelo de Reciprocidad de la Retroalimentación de las Respuestas a la Música, entendiendo la interacción en ellas de tres factores determinantes de estas respuestas: música, situación de escucha y escucha. De esta forma, los oyentes crean sus propias redes asociativas personales que actúan como puntos de referencia para sus representaciones mentales de sus mundos musicales.

Considera tres tipos de red, basados en las tres determinantes principales de respuestas a la música (Hargreaves, Hargreaves, \& North, 2012). El primer tipo es el propio de las piezas musicales a nivel estructural propio de cada persona, teniendo gran importancia "todas las experiencias musicales anteriores de ese individuo, toda la música que ha escuchado, que se recoge y almacena en el cuerpo y la mente de esa persona" (Hargreaves et al., 2012, p. 198). El segundo tipo, conecta las piezas de música con sus asociaciones culturales: situaciones típicas y contextos en los que se escuchan normalmente. En tercer lugar, las personas combinan sus redes de asociaciones musicales y culturales, incluyéndose aquí las asociaciones con personas, situaciones y eventos de sus vidas, por lo que son redes personales de asociación, sujetas a cambios frecuentes (Hargreaves, et al., 2012).

La experiencia y la abstracción del conocimiento musical en un nivel básico no parecen desarrollarse, por tanto, en un canal de comunicación pasivo (Cross, 2004), sino en 
un escenario mucho más rico y variado mediante acciones e interacciones participativas de todo tipo. Incluso la participación pasiva en la audición compromete la activación de regiones del cerebro que se vinculan al movimiento (Lakoff, \& Johnson, 1999; Janata, \& Grafton, 2003;). Será la correlación entre diferentes tipos de conocimiento básico en las interacciones de los individuos en entornos cotidianos, entre diferentes dominios de la experiencia y el dominio musical, lo que llevará a procesos metafóricos de aprehensión de significado gracias a la flexibilidad cognitiva y social que nos caracteriza como especie (Guck 1991; Zbikowsky, 1997, 1997-1998, 1998, 2002; Saslaw 1996, 1997-1998; Larson 1997; Brower 2000; Cross, 2004).

Así, el significado en general y el musical en particular, deben entenderse como fundados en la humanidad (Johnson, 1987): nuestra capacidad para construir significado, basada en las estructuras imaginativas por las que aprehendemos la realidad. Y ese significado que lo que atribuimos será básico para que la motivación lleve a un desencadenamiento de respuestas a lo escuchado, pero también a la inversa, la motivación hacia lo escuchado será lo que desencadene diferentes emociones en nosotros. Por lo tanto, motivación y emoción son cara y cruz de una misma moneda y solo pueden tener sentido en un contexto e individuo determinado. García, Martín, y Domínguez (2001) catalogan motivación y emoción como procesos activadores de cualquier proceso psicológico, por lo que uno, otro o ambos pueden desencadenar respuestas de acción conductual.

Desde esta relación, debe entenderse al individuo y su motivación relacionada con lo emocional y lo social como bases del aprendizaje constructivista, siendo todos estos aspectos primordiales en una disciplina artística y concretamente en el aprendizaje de la Música. Tanto es así que podemos decir que cualquier acción educativa debería estar basada en las asociaciones que se establecen entre estos constructos por ser inseparables, de lo que puede tomarse como ejemplo cualquiera de las investigaciones expuestas. Las investigaciones en Música sobre Motivación enfatizan, de una u otra forma, estas asociaciones, puesto que sus resultados aluden a ellas de forma considerable (Tabla 1).

\section{Conclusiones}

La Música es una de las principales actividades de ocio para niños, adolescente y jóvenes en sus diversas configuraciones, informales o formales. Esta población escucha música con regularidad, canta en coros, toca en bandas locales, y asiste a clases de instrumento; crea su propia música con grupos de amigos, imita a sus cantantes favoritos y habla de música con sus pares de música (Boal-Palheiros, 2004).

Algunos estudios han analizado las razones por las que los adolescentes oyen tanta música y las diferentes maneras que tienen de escucharla (Behne, 1997; Zillman, \& Gan, 1997; North, Hargreaves, \& O'Neill, 2000; Tarrant, North, \& Hargre aves, 2000). Otros, se han centrado en los usos y funciones que la música tiene para jóvenes y adultos en diferentes situaciones de la vida cotidiana (DeNora, 2000 Sloboda, O'Neill, \& Ivaldi, 2001).

Varias investigaciones demuestran la importancia capital que la música representa para los adolescentes y jóvenes de países occidentales (Larson, Kubey, \& CoIletti, 1989; Garton \& Pratt, 1991; Larson, 1995; Zillmann, \& Gan, 1997). La mayor parte de las razones por las que escuchan música hacen referencia a la satisfacción de necesidades emocionales y sociales, tales como: pasar el tiempo, aliviar el aburrimiento y la soledad, relajarse, hacer frente a los problemas, mejorar el estado de ánimo o divertirse (Gantz, Gartenberg, Pearson, \& Schiller, 1978; Roe, 1985 Brown, Campbell, \& Fischer, 1986; Fitzgerald, Joseph, Hayes, \& O'Reagan, 1995; Behne, 1997; Fernandes, Esteves, Dias, Lopes, Mendes, \& Azevedo, 1998; North et al., 2000; Tarrant et al., 2000).

Boal-Palheiros (2004) llevó a cabo un estudio sobre funciones y modos de oír música de niños y adolescentes de nacionalidades británica o portuguesa con rangos de edades: 9 y 10 años (escuelas primarias) y 13 y 14 (escuelas secundarias). Los resultados destacan los factores del desarrollo, la influencia de los contextos sociales y educativos, la funcionalidad de oír música, y la visión dinámica de los modos de oír de los niños. En casa y en contextos informales, la música tiene funciones emocionales y sociales, los niños desarrollan una identidad personal y social con ella, y disfrutan junto a familiares y amigos, con quienes mantienen relaciones emocionales y sociales significativas. En la escuela y otros contextos formales, 
parece tener sobre todo funciones cognitivas menos valoradas por los niños, y las relaciones sociales con compañeros y amigos se limitan a la convivencia. Los niños son conscientes de las diferencias entre estos contextos y atribuyen a las funciones sociales y emocionales gran importancia, pudiendo ser ésta una de las razones por las que disfrutan menos en la escuela.

Desde muy temprana edad, los niños se encuentran relacionados y bajo la influencia de diferentes grupos (escuela o grupo-clase: Arriaga, 2006 y familia: Cremades \& Lorenzo, 2007). Los grupos pueden moldear al niño desde el punto de vista psicológico, pero este estamento ya ha sido con anterioridad moldeado por la sociedad (de la Ossa, 2011), especialmente en las primeras etapas del desarrollo evolutivo (Torrico, 2012) y teniendo repercusiones en el propio desarrollo musical (Szubertowska, 2005; McPherson, 2009).

La creciente importancia de la música en la vida de los niños contrasta con el bajo impacto que las lecciones de música parecen tener sobre ellos (Lawson, Plummeridge, \& Swanwick, 1994; Mills, 1994; Ross, 1995; Gammon, 1996). Los maestros hacen preguntas sobre el repertorio más adecuado para motivar a los niños a escuchar, aprender y disfrutar de la Música. Por lo tanto, comprender la importancia que adquiere para los niños su participación en experiencias musicales informales sin duda puede beneficiar a la educación musical (Boal-Palheiros, 2004).

De todo lo expuesto, proponemos para la innovación en Música la necesidad inexcusable de una Metodología Constructivista que pueda albergar cualquier actividad en cualquier ámbito. En ella, motivación, emoción y entorno estarán presentes sin lugar a dudas.

Así, por ejemplo, deberá atenderse en ellas a la relación entre género musical y género del estudiante (Welch, Papageorgi, Haddon, Creech, Morton, de Bézenac, Duffy, Potter, Whyton, \& Himonides, 2008), expectativas y clima de trabajo (Hardré, \& Cox, 2009), las particularidades y características de alumnos que presentan diferencias con respecto a la media (Koshy, Pinherio-Torres, \& Portman-Smith, 2012), las diferencias en edades de aprendizaje (Nutbrown, 2013), el desarrollo de metodologías creativas propias de la disciplina (Hall, \& Thomson, 2017), las actitudes de las familias hacia el aprendizaje en diferentes momentos (West, Davies, \& Scott, 1992), o la construcción de relaciones en contextos determinados (Hughes, 2014). Desde el estudio bibliográfico presentado se puede afirmar su importancia para la educación musical, que debe ser ya entendida como prioridad y principio de innovación.

\section{Referencias bibliográficas}

Achtziger, A., \& Collwitzer, M. (2008) Motivation and volition in the course of action. En J. Heckhausen y $\mathrm{H}$. Heckhausen (Eds.). Motivation and action (pp. 272-295). New York: Cambridge University Press.

Arriaga, C. (2006). Importancia de los condicionantes contextuales en la educación musical. Música y Educación, 19(68), 131-140.

Austin, J. R. \& Haefner, M. H. (2006). Exploring music practice among sixth-grade band and orchestra students. Psychology of Music, 34(4), 535-558. doi 10.1177/0305735606067170

Behne, K. E. (1997). The development of 'Musikerleben' in adolescence: how and why young people listen to music. En
I. Deliège \& J. Sloboda (Eds.), Perception and cognition of music (pp.143-159). East Sussex: Psychology Press.

Boal-Palheiros, G. (2004). Funciones y modos de oír música de niños y adolescentes en distintos contextos. Revista de Psicodidáctica, 17, 5-25.

Brower, C. (2000). A cognitive theory of musical meaning. Journal of Music Theory 44(2), 323-379.

Brown, J. D., Campbell, K. \& Fischer, L. (1986). American adolescents and music videos: Why do they watch? Gazette, 37, 19-32. doi 10.1177/001654928603700104

Candelario, M. E. (2009). Las emociones en un contexto de innovación educativa. Reduca, 1(2), 596-607.

Casas-Mas, A. (febrero, 2008). Una revisión crítica sobre las metodologías de investigación en enseñanza y aprendizaje de la interpretación musical: propuestas de mejora. Trabajo presentado en el I congreso de educación e investigación musical. Metodologías aplicadas y enfoques pedagógicos en la enseñanza musical de la Universidad Autónoma de Madrid, Madrid. 
Collier, G. L. (2007). Beyond valence and activity in the emotional connotations of music. Psychology of Music, 35(1), 110-131. doi 10.1177/0305735607068890

Creech, A., \& Hallam, S. (2009). Interaction in instrumental learning the influence of interpersonal dyna mics on parents. International Journal of Music Education, 27(2), 94-106. do $10.1177 / 0255761409102318$

Cremades, R., \& Lorenzo, O. (2007). Familia, música y educación informal. Música y Educación, 20(72), 35-46.

Cremades, R., Herrera, L., \& Lorenzo, O. (2011). Las motivaciones de los niños para aprender música en la escuela de música y danza de Melilla. DEDiCA. Revista de Educacao e Humanidades, 1, 293-318.

Cross, I. (2004). Music, meaning, ambiguity and evolution. En D. Miell, R. Mac Donald \& D. Hargreaves (Eds). Musical Communication (pp. 27-43). Oxford Oxford University Press.

de la Ossa, M. A. (2011). Sociología de la música: relación con la educación musical. Música y Educación, 24(87), 18-35.
DeNora, T. (2000). Music in everyday life. Cambridge: Cambridge University Press. doi https://doi.org/10.1017/ CBO9780511489433

Elliot, A. J. (2008). Approach and avoidance motivation. En A. J. Elliot (Ed.) Handbook of approach and avoidance motivation (pp. 3-14). New York: Psychology Press.

Fernandes, A. T., Esteves, A. J., Dias, I., Lopes, J. T., Mendes, M. M. \& Azevedo, N. (1998). Práticas e aspirações culturais. Os estudantes da cidade do Porto. Porto: Edições Afrontamento e Câmara Municipal do Porto.

Fitzgerald, M., Joseph, A. P., Hayes, M., \& O'Reagan, M. (1995). Leisure activities of adolescent children. Journal of Adolescen$c e, 18,349-358$

Gammon, V. (1996). What is wrong with school music? A response to Malcolm Ross. British Journal of Music Education, 12(3), 101-122. doi https://doi. org/10.1017/S0265051700003089

Gantz, W., Gartenberg, H., Pearson, M., \& Schiller, S. (1978). Gratifications and expectations associated with popular music among adolescents. Popular Music and Society, 6(1), 81-89. doi http://dx.doi. org/10.1080/03007767808591113

García, E., Martín, M. D., \& Domínguez, J. (2001). Procesos Psicológicos. Madrid: Ediciones Pirámide.

Garton, A. F. \& Pratt, C. (1991). Leisure activities of adolescent school students: predictors of participation and interest. Journal of Adolescence, 14(3), 305-321. doi http://dx.doi.org/10.1016/0140-1971(91)90023-K

Ghazali, G. (2006). Factors influencing malaysian children's motivation to learn music (Phd Thesis). Music \& Music Education, Faculty of Arts \& Social Sciences, Univesity of New South Wales, South Wales.

Guck, M. (1991) Two types of metaphoric transfer. En J.C. Kassler (Ed) Metaphor a musical dimension (pp.1-12). Sydney: The Currency Press.

Hall, Ch. \& Thomson, P. (2017). Creativity in teaching: what can teachers learn from artists? Research Papers in Education, 32(1), 106-120. doi http://dx.doi.org/10. 080/02671522.2016.1144216
Hallam, S. (2001). The development of metacognition in musicians: Implications for education. British Journal of $\mathrm{Mu}$ sic Education, 18(1), 27-39. doi https://doi. org/10.1017/S0265051701000122

Hallam, S. (2002). Musical motivation: Towards a model synthesising the research. Music Education Research, 4(2), 225-244. doi http://dx.doi. org/10.1080/1461380022000011939

Hallam, S. (2013). What predicts level of expertise attained, quality of performance, and future musical aspirations in young instrumental players? Psychology of Music, 41(3), 267-291. do $10.1177 / 0305735611425902$

Hallam, S., Varvarigou, M., Creech, A., Papageorgi, I., Gomes, T., Lanipekun, J., \& Rintal, T. (2017). Are there gender differences in instrumental music practice? Psychology of Music, 45(1), 116-130. do 10.1177/0305735616650994

Hallam, S., Rogers, L., \& Creech, A (2008).Gender differences in musical instrument choice. International Journal of Music Education, 26(1), 7-19. do $10.1177 / 0255761407085646$ 
Hardré, P. \& Cox, M. (2009). Evaluating faculty work: expectations and standards of faculty performance in research universities. Research $\mathrm{Pa}$ pers in Education, 24(4), 383-419. doi $10.1080 / 02671520802348590$.

Hargreaves, D. J., Hargreaves, J., \& North, A. (2012). Imagination and creativity in music listening. En D.J. Hargreaves, D.J.E. Miell, \& R.A.R. MacDonald (Eds.), Musical imaginations (pp. 156-172). Oxford, UK: Oxford University Press.

Heckhausen, J., \& Heckhausen, $H$. (2008). Motivation and action: Introduction and overview. En J. Heckhausen y $\mathrm{H}$. Heckhausen (Eds.), Motivation and action. New York: Cambridge University Press.

Hernández-Pina, F. (2001). La Calidad de la Enseñanza y el Aprendizaje en Educación Superior. Revista de investigación educativa, 19(2), 465-489.

Herrera, L., \& Cremades, R. (2011). Gus$\infty \quad$ tos musicales de los estudiantes de conservatorio. Música y Educación, 24(85), 6476. mind. Chicago: The University of Chicago Press.
Johnson, M. (1987). The body in the

Herrera, L., \& Oya, L. (2014). Causas de elección instrumental: un estudio en los conservatorios profesionales de Málaga capital. Eufonía. Didáctica de la Música, 62, 43-54.

Holgado, F. P., Navas, L., \& Marco, V. (2013). The Students' Academic Performance at the Conservatory of Music: A Structural Model from the Motivational Variables. Revista de Psicodidáctica, 18(2), 257-273. doi 10.1387/RevPsicodidact.6942

Hughes, J. (2014). Contact and context: sharing education and building relationships in a divided society. Research Papers in Education, 29(2), 193-210. doi http://dx. doi.org/10.1080/02671522.2012.754928

Janata, P., \& Grafton, S. T. (2003). Swinging in the brain: shared neural substrates for behaviors related to sequencing and music. Nature neuroscience, 6, 682687. doi 10.1038/nn1081
Juslin, P. (1996). Emotional Expression in Music Performance: Between the Performer's Intention and the Listener's Experience. Psychology of Music, 24(1), 6891. doi 10.1177/0305735696241007

Juslin, P. (1997a). Can Results from Studies of Perceived Expression in Musical Performances Be Generalized across Response Formats? Psychomusicology, 16(12), 77-101. doi http://dx.doi.org/10.1037/ h0094065

Juslin, P. (1997b). Emotional Communication in Music Performance: A Functionalist Perspective and Some Data. Music Perception, 14(4), 383-418. doi $10.2307 / 40285731$

Juslin, P. (1997c). Perceived Emotional Expression in Synthesized Performances of a Short Melody: Capturing the Listener's Judgment Policy. Musicae Scientiae 1(2), 225-256. doi $10.1177 / 102986499700100205$

Kinney, D.W. (2010). Selected Nonmusic Predictors of Urban Students' Decisions to Enroll and Persist in Middle School Band Programs. Music Education Research, 57(4), 334-350. doi 10.1177/0022429409350086
Koshy, V., Pinherio-Torres, C., \& Portman-Smith, C. (2012). The landscape of gifted and talented education in England and Wales: how are teachers implenenting policy? Research Papers in Education, 27(2), 167-186. doi http://dx.doi.org/10.1 080/02671522.2010.509514

Lakoff, G., \& Johnson, M. (1999). Philosophy in the Flesh. The Embodied Mind and its Challenge to Western Thought. New York: Basic Books.

Larson, S. (1997) The problem of Prolongation in "Tonal" Music: Terminology, Perception, and Expressive Meaning. Journal of Music Theory, 41(1), 101-136.

Larson, R. (1995). Secrets in the bedroom. Adolescents private use of media. Journal of Youth and Adolescence, 24(5), 535-550. doi 10.1007/BF01537055

Larson, R.W., Kubey, R., \& Colletti, J. (1989). Changing channels: early adolescent media choices and shifting investments in family and friends. Journal of Youth and Adolescence, 18(6), 583-599. doi 10.1007/BF02139075 
Lawson, D., Plummeridge, C., \& Swanwick, K. (1994). Music and the National Curriculum in primary schools. British Journal of Music Education, 11(1), 3-14. doi https://doi.org/10.1017/ S0265051700001996

León, J., Nuñez, J. L., Zuleica, R. A., \& Bordón, B. (2015). Music Academic Performance: Effect of Intrinsic Motivation and Critical Thinking Revista de Psicodidáctica, 20(2), 377-391. doi 10.1387/RevPsicodidact. 12673

Maclntyre, P., Potter, G., \& Burns, J. (2012). The socio-educational model of music motivation. Journal of Research in Music Education, 6(2), 129-144. doi $10.1177 / 0022429412444609$

Marco, V. (2010). El rendimiento académico en el conservatorio de música: análisis de variables motivacionales (Tesis doctoral). Universidad de Alicante, Alicante.

McClelland, D. C. (1985). Estudios de la motivación humana. Madrid: Narcea.

McPherson, G. E. (2009). The role of parents in children's musical development. Psychology of Music, 37(1), 91-110. doi $10.1177 / 0305735607086049$
McPherson, G. E., Osborne, M. S., Barrett, M. S., Davidson, J. W., \& Faulkner, R. (2015). Motivation to study music in Australian schools: The impact of music learning, gender, and socio-economic status. Research Studies in Music Education, 37(2), 141-160. doi 10.1177/1321103X15600914

Meyer, D. K., \&Turner, J. C. (2002). Discovering Emotion in Classroom Motivation Research. Educational Psychologist, 37(2), 91-106. doi http://dx.doi.org/10.1207/ S15326985EP3702_5

Millican, J. Si (2016). Describing Preservice Instrumental Music Educators' Pedagogical Content Knowledge. Applications of Research in Music Education, 34(2), 6168. doi 10.1177/8755123314552664

Mills, J. (1994). Music in the National Curriculum. The first year. British Journal of Music Education, 11(3), 191-196. doi https://doi.org/10.1017/ S0265051700002151

Montero, I., \& Huertas, J. A. (2003). Técnicas de medida y métodos de investigación de la motivación. En E. Fernández Abascal, P. Jiménez y M. D. Martín (Eds.). Emoción y motivación. Madrid: Centro de Estudios Ramón Areces.
Navas, L., Iborra, G., \& Sampascual, G. (2007). Las metas académicas de los estudiantes de ESO en la clase de Música. Revista de Psicodidáctica, 12(1), 131-142.

North, A. C., Hargreaves, D. J., \& O'NeiII, S. (2000). The importance of music to adolescents. British Journal of Edu cational Psychology, 70(2), 255-272. doi $10.1348 / 000709900158083$

Nutbrown, C. (2013). Conceptualising arts-based learning in the early years. $R e-$ search Papers in Education, 28(2), 239-263. doi http://dx.doi.org/10.1080/02671522. 2011.580365

Reeve, J. (1992). Motivación y emoción. Madrid: McGraw-Hill.

Renwick, J. M. (2008). Because l love playing my instrument: young musicians' internalised motivation and self-regulated practising behavior (Tesis doctoral). Media, \& Performing Arts, Faculty of Arts \& Social Sciences, University of New South Wales University, South Wales.
Rodríguez, M. (2009) Motivar para aprender en situaciones académicas. En G. Romero y A. Caballero (Eds.). La crisis de la escuela educadora (pp. 207-242). Barcelona: Laertes.

Roe, K. (1985). Swedish youth and music: Listening patterns and motivations Communication Research, 12(3), 353-362. doi 10.1177/009365085012003007

Ross, M. (1995). What's wrong with school music? British Journal of Music Education, 12(3), 185-201. doi https://doi. org/10.1017/S0265051700002692

Saslaw, J. (1996). Forces, Containers, and Paths: The Role of Body-Derived Image Schemas in the Conceptualization of Music. Journal of Music Theory, 40(2), 217 243.

Saslaw, J. (1997-1998). Life Forces: Conceptual Structures in Schenker's "Free Composition" and Schoenberg's "The Musical Idea". Theory and Practice, 22-23, 17 33. 
Schutz, P. A., \& DeCuir, J. T. (2002). Inquiry on Emotions in Education. Educational Psychologist, 37(2), 125-135. doi http:// dx.doi.org/10.1207/S15326985EP3702_7

Sloboda, J. A., O'Neill, S., \& Ivaldi, A. (2001). Functions of Music in Everyday Life: AnExploratoryStudyUsing the Experience Sampling Method. Musicae Scientia, 5(1), 9-29. doi 10.1177/102986490100500102

Sloboda, J. (2012). La mente musical: La psicología cognitiva de la música. Madrid: Machado.

StGeorge, J., Holbrook, A., \& CantweII, R. (2014). Affinity for music: A study of the role of emotion in musical instrument learning. International Journal of MusicEducation, 32(3), 264-277. do $10.1177 / 0255761413491178$

Szubertowska, E. (2005). Education and the music culture of Polish adolescents. Psychology of Music, 33(3), 317-330. doi 10.1177/0305735605053736

Tarrant, M., North, A. C., \& Hargreaves, D. J. (2000). English and American adolescents' reasons for listening to music. Psychology of Music, 28(2), 166-173.
Thrash, T. M., \& Hurst, A. (2008) Approach and avoidance motivation in the achievement domain: Integrating the achievement motive and achievement goal tradition. En A. J. Elliot (Ed.), Handbook of approach and avoidance motivation (pp. 217-233). New York: Psychology Press.

Torrico, R. (2012). Niños musicales: autodidactas del siglo XXI. Música y Educación, 25(90), 16-28.

Vargas, L. M. (1994). Sobre el concepto de percepción. Alteridades, 4(8), 47-53.

Vasil, M. (2013).Extrinsic Motivators Affecting Fourth-Grade Students' Interest and Enrollment in an Instrumental Music Program. Applications of Research in Music Education, 32(1), 74-82. doi $10.1177 / 8755123313502345$

Welch, G., Papageorgi, I., Haddon, L., Creech, A., Morton, F., de Bézenac, C.,... \& Himonides, E. (2008). Musical genre and gender as a factor in higher education learning in music. Research Papers in Education, 23(2), 203-217. doi http://dx.doi. org/10.1080/02671520802048752
West, A., Davies, J. \& Scott, G. (1992). Attitudes to secondary school: parents' views over a five-year period. Research $\mathrm{Pa}$ pers in Education, 7(2), 129-149. doi http:// dx.doi.org/10.1080/0267152920070203

Zbikowsky, L. M. (1997). Conceptual Models and Cross-Domain Mapping: New Perspectives on Theories of Music and Hierarchy. Journal of Music Theory, 41(2), 193-225.

Zbikowsky, L. M. (1997-1998). Des Herzraums abschied: Mark Johnsotú theory of embodied knowledge and music theory. Theory and Practice, 22-23, 1-16.

Zbikowsky, L. M. (1998). Metaphor and music theory: reflections from cognitive science. Music Theory Online, 4(1).

Zbikowsky, L. M. (2002). Conceptualizing Music: Cognitive Structure, Theory, and Analysis. Oxford: Oxford University Press.

Zillman, D., \& Gan, S. (1997). Musical taste in adolescence. En D. J. Hargreaves \& A. C. North (Eds.), The social psychology of music (pp. 161-187). Oxford: Oxford University Press. 